\title{
Compersto
}

\section{Competência em Informação: estudos introdutórios sobre a temática na Itália}

\author{
Marcela Reinhardt de Souza \\ Mestra; Universidade do Estado de Santa Catarina, Florianópolis, SC, Brasil; \\ marcela.reinhardt@yahoo.com.br \\ Elizete Vieira Vitorino \\ Doutor; Universidade Federal de Santa Catarina, Florianópolis, SC, Brasil; \\ elizete.vitorino@ufsc.br
}

\begin{abstract}
Resumo: Este estudo aborda o tema da information literacy na Itália. Com aumento do número de informações disponíveis para a população, o desenvolvimento da competência em informação ganha importância na atual sociedade. Este artigo identifica a literatura publicada sobre competência em informação na Itália, por meio de uma pesquisa bibliográfica. Aponta as fontes de informação com trabalhos disponíveis sobre a temática; identifica as principais publicações e verifica as temáticas apresentadas nas publicações científicas a respeito da competência em informação na Itália. Foram recuperados documentos nas bases de dados Directory of Open Access Journals, Library and Information Science Abstracts, Information Science \& Technology Abstracts e Web of Science e nos levantamentos publicados por Basili (2014) e Crocetti (2012). Os resultados foram complementados com dados obtidos na European Conference on Information Literacy dos anos de 2013 a 2018, o que perfez 136 documentos. Verificou-se crescente debate na área a partir dos anos 1990, que se intensifica após 2004 com pesquisadores italianos influentes como Carla Basili e Laura Ballestra. Os estudos italianos identificados relacionavam a competência em informação com diversos temas, entre eles: a preocupação com políticas públicas inexistentes ou insuficientes, ações práticas, questões éticas e ambientais, grupos vulneráveis, a área da saúde e tecnologia.
\end{abstract}

Palavras-chave: Competência em informação. Revisão bibliográfica. Itália.

\section{Introdução}

A informação na sociedade atual recebe lugar de destaque. Circula com velocidade e é criada em grande quantidade e por isso tornou-se "[...] uma fonte vital para as economias mundiais e certamente é o componente básico da educação. [...] Representa também diferentes desafios para pessoas de qualquer condição [...]" (LAU, 2007, p. 6). O desafio a que se refere Lau (2007) está na 
dificuldade em avaliar a informação e apurar quais são válidas de acordo com a necessidade de cada situação.

Com o desenvolvimento da tecnologia e sua popularização, a informação passou a estar disponível em grande quantidade para as pessoas. Nesse sentido, Caregnato (2000) já afirmava que, à medida que surgem novas formas de acessar a informação digital em rede, são necessárias novas e "[...] mais aprimoradas habilidades para buscar, selecionar, sintetizar e utilizar estas informações [...]" (CAREGNATO, 2000, p. 53). É nesse sentido que se iniciam os estudos da information literacy, termo que traduziremos aqui por competência em informação, expressão amplamente difundida no cenário brasileiro e que no mundo se traduz em variadas formas ${ }^{1}$.

Basili (2008b), preocupada em estudar a temática no contexto italiano, faz duas definições de information literacy. A primeira definição é de information literacy como processo " $[\ldots]$ educativo, político, que visa disseminar em uma população um nível mínimo de competência para a recuperação, avaliação e exploração da informação a partir de uma variedade de fontes.” (BASILI, 2008b, p. 36, tradução nossa). A segunda definição é de information literacy como status: "[...] finalidade social da política educacional; estado ou condição oriundos de um processo e pessoa que adquiriu a competência para recuperar, avaliar e utilizar as informações a partir de uma variedade de fontes." (BASILI, 2008b, p. 36, tradução nossa).

A discussão da temática da competência em informação se apresenta em constante avanço ao redor do mundo. Cada vez mais pesquisadores, de diferentes países e culturas, se ocupam em investigar as bases deste assunto e aplicá-las aos seus respectivos cenários. Nesta perspectiva, reconhecer as trajetórias da competência em informação na Itália foi o que nos preocupou neste trabalho. Tal como em outros países europeus, a produção italiana no que concerne ao tema é significativa e segue a passos firmes em busca de reconhecimento.

A maior parte do que trazemos neste trabalho se refere ao levantamento de publicações sobre a competência em informação na Itália a partir do que está disponível em bases de dados do Portal de Periódicos da CAPES e em 
levantamentos realizados até o ano de 2019 sobre o assunto em diversas outras fontes disponíveis online.

Esta pesquisa busca apresentar ao leitor interessado na temática investigada naquele país o quanto já foi pesquisado e publicado sobre a competência em informação. Segue-se a isso o fato de que o modo como a produção científica na área se apresenta em outros contextos é relevante para que o investigador brasileiro possa avançar nas pesquisas e ações sobre o assunto, ao tomar conhecimento de outras realidades. Tratar desse tema em outros contextos que não o do Brasil e a partir da experiência e da vivência de investigadores de outros contextos pode favorecer o surgimento de ideias em torno do debate, da implantação de projetos e de ações para a competência em informação.

Com vistas a alcançar o propósito maior da pesquisa, seguem-se três objetivos específicos: o primeiro deles é apontar as fontes de informação nas quais há trabalhos disponíveis sobre competência em informação na Itália; o segundo é identificar as principais publicações sobre o tema, o que se alcançou a partir de estratégias de busca aplicadas às buscas nas fontes de informação encontradas; o terceiro, por sua vez, é identificar quais são as temáticas apresentadas nesses documentos, o que se atingiu com a seleção, avaliação e leitura dos documentos pertinentes ao assunto encontradas nas fontes de informação.

Para dar conta destes objetivos, inicialmente trataremos de esclarecer os procedimentos metodológicos adotados no estudo bibliográfico sob alguns aspectos e, a seguir, apresentaremos os resultados obtidos na investigação, trazendo à tona a competência em informação sob o olhar italiano.

\section{Procedimentos metodológicos}

Com base nos objetivos a serem atingidos, esta pesquisa é exploratória, pois visa determinar o que já foi publicado sobre competência em informação na Itália. Corresponde à pesquisa bibliográfica por meio de levantamentos em bases de dados e outros levantamentos bibliográficos publicados anteriormente. 
A fim de atender aos objetivos da pesquisa, foram realizadas buscas nas seguintes bases de dados: Directory of Open Access Journals (DOAJ), Library and Information Science Abstracts (LISA), Information Science \& Technology Abstracts (ISTA) e Web of Science. Foi utilizado o Portal de Periódicos da Coordenação de Aperfeiçoamento de Pessoal de Nível Superior (CAPES) para encontrar as bases, com a busca: Áreas do Conhecimento=Ciências Sociais Aplicadas, Subcategoria=Ciência da Informação. Dos 88 resultados, 18 contêm referenciais com resumos. Buscaram-se fontes internacionais, que pudessem fornecer diferentes resultados, considerando que houvesse ao menos uma de acesso aberto e as demais de empresas diferentes.

Essas bases foram selecionadas a partir dos indicadores de Tomaél, Alcará e Silva (2016). De acordo com as autoras, esses indicadores são proposições para analisar uma fonte de informação a partir de variáveis, que têm critérios para auxiliar na tomada de decisão (TOMAÉL; ALCARÁ; SILVA, 2016). Os indicadores selecionados foram: aspectos extrínsecos, aspectos intrínsecos e aspectos contextuais. Quanto aos aspectos extrínsecos, o critério utilizado foi a usabilidade, relacionada à facilidade de navegação, funcionalidade e estrutura da informação. Os aspectos intrínsecos envolveram os critérios de precisão, consistência e relevância, atualização, integridade e alcance. Para os aspectos contextuais, os critérios foram a conveniência, estabilidade e adequação.

Para o acesso aberto, a DOAJ foi selecionada por abranger um número maior de periódicos científicos. Essa base tem critérios rígidos de indexação, mas não tem limite de aceite por área do conhecimento. As demais bases representam empresas diferentes; ISTA da ProQuest, LISA da EBSCO e Web of Science da Clarivate Analytcs.

Os termos de busca utilizados foram "competenza informativa" e "information literacy" AND "Italy". Os idiomas selecionados para comporem os resultados foram inglês, italiano e português; a data-limite utilizada foi 30 de setembro de 2019 e as buscas foram realizadas durante o mês de outubro de 2019. 
Além das buscas nas bases de dados supracitadas, também foram utilizados os levantamentos de Basili (2014) e Crocetti (2012), dos quais foram selecionados trabalhos relacionados ao tema para utilização neste artigo e trabalhos apresentados nas diferentes edições da European Conference on Information Literacy (ECIL), entre os anos de 2013 a 2018.

As buscas recuperaram 136 documentos. A escolha destes documentos ocorreu porque eram pesquisas de interesse para o estudo, que foram desenvolvidas na Itália e que tratavam da temática da competência em informação. Inicialmente, foram analisados os títulos, as palavras-chave e os resumos dos artigos recuperados. Com isso, aqueles considerados relevantes foram utilizados para compor o referencial teórico deste trabalho.

\section{Competência em informação na Itália}

Por meio do levantamento da produção científica da Itália, é possível perceber que a competência em informação foi e é estudada em diferentes contextos pelos pesquisadores italianos e que a necessidade de programas voltados à competência em informação com ações práticas é mencionada nos artigos publicados. Constata-se também que diversos níveis de pesquisa foram desenvolvidos com foco em contextos distintos.

Por meio do quadro a seguir, é possível visualizar os resultados do levantamento bibliográfico obtido a partir dos trabalhos de Basili (2014) e de Crocetti (2012), das bases de dados DOAJ, LISA e Web of Science, ISTA e da ECIL.

Tabela 1 - Competência em informação na Itália, segundo segundo Basili (2014) e Crocetti (2012), a ECIL e levantamentos nas bases de dados DOAJ, LISA, ISTA e Web of Science.

\begin{tabular}{|c|c|c|c|c|c|c|c|}
\hline \multirow{2}{*}{ Ano } & \multicolumn{7}{c|}{ Fonte } \\
\cline { 2 - 8 } & $\begin{array}{c}\text { Basili } \\
(\mathbf{2 0 1 4})\end{array}$ & $\begin{array}{c}\text { Crocetti } \\
\mathbf{( 2 0 1 2 )}\end{array}$ & ECIL & DOAJ & LISA & $\begin{array}{c}\text { Web of } \\
\text { Science }\end{array}$ & ISTA \\
\hline 1995 & - & - & - & - & 1 & - & - \\
1998 & 1 & - & - & - & - & - & - \\
1999 & 4 & - & - & - & 2 & - & - \\
2000 & - & - & - & - & - & - & - \\
2001 & 2 & - & - & - & - & - & - \\
2002 & - & - & - & - & - & - & - \\
2003 & 2 & - & - & - & 2 & - & - \\
\hline
\end{tabular}




\begin{tabular}{llllllll}
2004 & 10 & - & - & - & - & - & - \\
2005 & 2 & - & - & - & - & - & - \\
2006 & 3 & 1 & - & - & 2 & - & - \\
2007 & 2 & 2 & - & - & 2 & - & - \\
2008 & 3 & 6 & - & - & 1 & - & - \\
2009 & 2 & 4 & - & - & - & - & - \\
2010 & 3 & 6 & - & 1 & - & - & - \\
2011 & 6 & 6 & - & - & 2 & - & - \\
2012 & 2 & 6 & - & - & - & - & - \\
2013 & - & - & 6 & 5 & - & - & - \\
2014 & - & - & 4 & 5 & - & 1 & - \\
2015 & - & - & 4 & 1 & - & - & 1 \\
2016 & - & - & 3 & 1 & 1 & 3 & 1 \\
2017 & - & - & 3 & 3 & 1 & - & - \\
2018 & - & - & 2 & 1 & - & 2 & - \\
$2019 *$ & - & - & - & - & - & 2 & - \\
\hline
\end{tabular}

Fonte: Dados da pesquisa e nos levantamentos de Basili (2014) e Crocetti (2012).

Nota: * Até 30 de setembro do referido ano.

Observa-se, por meio da literatura investigada, que o DOAJ é, entre as bases de dados, a que apresenta o maior número de documentos sobre competência em informação na Itália. Por outro lado, ao analisar a relevância dos documentos recuperados dentro do tema desta pesquisa, Basili (2014) apresenta-se como uma das autoras que mais publicou sobre o assunto. Nota-se que as pesquisas sobre o tema tiveram início na segunda metade da década de 1990 e se intensificaram a partir de 2004. Ao todo foram identificadas 136 publicações, divulgadas principalmente em revistas como Biblioteche Oggi, AIB Notizie e AIDAInformazioni.

É fato que a competência em informação é um tema amplamente estudado na Itália. Foram recuperados dois levantamentos específicos a respeito desses estudos que auxiliaram nesta pesquisa. Crocetti (2012) publicou no site oficial da região da Toscana, na Itália, uma bibliografia sobre competência em informação sob o título de Information literacy per ragazzi: come favorire la competenza informativa ${ }^{2}$. Horton Junior (2014) elaborou um levantamento dos documentos já publicados sobre competência em informação em diversos idiomas e Carla Basili (2014) foi a responsável por identificar os documentos disponíveis em língua italiana. Basili (2014) apresenta 42 documentos publicados entre os anos de 1998 e 2012, por meio de uma lista organizada por 
data de publicação, a fim de possibilitar reflexões acerca do desenvolvimento das pesquisas no campo da competência em informação.

O foco inicial de algumas pesquisas precursoras sobre a competência em informação consistiu no treinamento de usuários. Boccardi e Zappa (1995) destacaram a importância de considerar as especificidades e a autonomia das realidades de cada biblioteca escolar, para que fosse elaborado um projeto de estratégias de pesquisa e seleção para ser implantado nesses espaços. $\mathrm{O}$ enfoque dado pelos autores está na biblioteca escolar, no treinamento de usuários e na importância do bibliotecário nessas unidades de informação; não há utilização explícita do termo information literacy, apesar de o estudo tratar sobre o tema. Tammaro (1999) também versa a respeito da importância do treinamento de usuários e exprime a crença de que esse seria um serviço ampliado e transformado com o tempo, tanto que, em países como os Estados Unidos, já se percebia a função de educar os usuários quanto à correta utilização da biblioteca e das fontes bibliográficas, sendo essas ações chamadas de information literacy.

A primeira publicação citada por Basili (2014) sobre competência em informação é de 1998 e foi publicada por Maria Pia Carosella (1998) na AIDA Informazioni: Rivista di Scienze dell'informazione ${ }^{3}$. Carosella (1998) trata do desenvolvimento das "competenze di informazione", como traduz o termo information literacy, e se refere ao projeto Develop European Competencies in Information and Documentation (DECID), apresentado pela Association des professionnels de l'information et de la documentation (ADBS), organização francesa. De acordo com Carosella (1998), o DECID visava definir as tarefas europeias para competência em informação com base nas implementações existentes; desenvolver uma certificação europeia para a competência, a partir dos sistemas de certificação em vigor em países europeus e desenvolver, em nível europeu, mecanismos de formação continuada que permitissem o desenvolvimento de novas habilidades exigidas ao novo profissional da informação.

Nem todos os autores italianos optaram por traduzir o termo information literacy. Tammaro (1999) mantém o termo utilizado naquele momento nos Estados Unidos, definido como “[...] função de educar o usuário ao uso correto 
da biblioteca e das fontes bibliográficas [...]" (TAMMARO, 1999, p. 46). A autora ainda destaca que um cidadão na sociedade da informação precisa saber usar a informação e que naquele momento os bibliotecários italianos não estavam cientes da importância dessa atividade, apontada como um novo papel para as bibliotecas (TAMMARO, 1999).

Pensando nos profissionais da informação, Carosella (1999) trata do Euroguida di riferimento I \& $D$ dei professionisti europei dell'informazione e documentazione $e^{4}$. O documento reflete a intenção presente naquela ocasião de elaborar uma certificação para os profissionais da informação, de acordo com uma avaliação de suas capacidades.

A visão de que é importante trabalhar a competência em informação no mundo empresarial também está presente na literatura italiana sobre o tema. Carla Basili (1999) divulgou a ocorrência do evento intitulado La Cultura dell'Informazione: opportunità e strategie ${ }^{5}$. O objetivo deste foi sensibilizar as empresas

[...] sobre os valores estratégicos da informação, evidenciando a oportunidade de um processo de alfabetização dos profissionais aos temas de tratamento e uso dos diversos tipos de fontes e múltiplos serviços/instrumentos. (BASILI, 1999, p. 11, tradução nossa).

A existência de cursos em bibliotecas com foco na competência em informação é observada por Fjällbrant (1999). No entanto, o que chamou atenção do autor foi o crescimento de cursos a distância. $O$ autor supracitado verifica que muitos alunos de cursos não presenciais tinham dificuldade em lidar com as tecnologias para encontrar a informação da qual necessitavam (FJÄLLBRANT, 1999). Com isso, o autor sugere que as bibliotecas acadêmicas e públicas deveriam oferecer programas de formação a distância para os usuários (FJÄLLBRANT, 1999). Como ainda não há consenso na tradução do termo para o italiano, Fjällbrant (1999) traduz o termo information literacy como alfabetizzazione all'uso delle informazioni ${ }^{6}$.

Basili (2001) traduziu de maneira inusitada o termo information literacy como cultura dell'informazione ${ }^{7}$. Basili (2001) apresenta uma proposta de intervenções para que a Itália promova a cultura dell'informazione: sensibilizar os vários estratos da sociedade pedindo ação legislativa; sensibilizar e formar os 
professores; projetar instrumentos de formação diferenciada de acordo com a idade escolar e instituir nas universidades cursos para o uso dos recursos informacionais. O termo em italiano competenza informativa já é utilizado em 2004 por Renditiso (2004) em sua publicação da International Federation of Library Associations (IFLA) no congresso em Buenos Aires, ocorrido no mesmo ano.

Mamoli e Gorreri (2003) especificam que information literacy é o termo utilizado nos Estados Unidos em referência às atividades de instrução ao uso de instrumentos bibliográficos, para tornar os usuários das bibliotecas mais autônomos a respeito de sua capacidade de "[...] buscar, selecionar, avaliar e organizar de modo competente e crítico os recursos informacionais." (MAMOLI; GORRERI, 2003, p. 22, tradução nossa). Para que um estudante seja autônomo no que se refere à informação, Mamoli e Gorreri (2003) apontam a necessidade de saber quando há "um problema de informação", de saber usar estratégias de busca, saber localizar e acessar a informação, saber selecionar e utilizar a informação, saber sintetizar e apresentar a informação e saber avaliar a informação.

O uso do e-learning no desenvolvimento da competência em informação é apresentado por Ballestra (2003) em estudo que contém resultados de pesquisa com usuários da Biblioteca Mario Rostoni da Universidade Carlo Cattaneo, de Castellanza. Ballestra (2003) indica que os usuários da biblioteca têm oportunidades diversas de aprendizado por meio da internet e da pesquisa bibliográfica e que o instrumento do e-learning é eficaz no desenvolvimento da competência em informação de usuários e de bibliotecários. A autora defende que a competência em informação permite aos usuários autonomia no complexo mundo informacional (BALLESTRA, 2003).

Basili (2004) destaca a ausência de uma política europeia em termos de cultura da informação (como a autora traduz information literacy), principalmente em comparação com os Estados Unidos, país no qual a autora afirma haver uma forte vontade política nesta direção. A autora apresenta o projeto European network on Information Literacy (EnIL), que visa a longo prazo "[...] desenvolver uma estratégia comum a nível europeu pela information 
literacy [...]" (BASILI, 2004, p. 48, tradução nossa). Basili (2008a) aponta que a information literacy é um fenômeno multidimensional e amplamente discutido na literatura com foco para bibliotecas e que o EnIL mantém o foco nas dimensões políticas e de pesquisa.

No entanto, Basili (2008a) afirma que o desenvolvimento de políticas públicas voltadas à information literacy na Europa está em fase inicial e há necessidade de incluir uma disciplina nos currículos do ensino superior europeu que trate especificamente da competência em informação. Basili (2008a) faz uma crítica ao fato de os decisores políticos parecerem desconhecer esta questão, ainda que ela tenha sido debatida desde os anos de 1970 no mundo. Virkus (2003) também destacou que de acordo com os resultados de sua pesquisa sobre a evolução da competência em informação na Europa, faltam iniciativas governamentais nesse sentido. Virkus (2003) identificou que as iniciativas em torno da competência em informação na Europa até 2003 ocorreram principalmente em ambientes escolares e acadêmicos e em instituições como a European Association of Distance Teaching Universities, The Nordic Council of Scientific Information e The Danish Union of Librariansalém das conferências e seminários, como a International Conference on Information Technology and Information Literacy, em 2002, na Escócia; Creating Knowledge, em 1999, na Suécia e outros eventos em países como Espanha, Finlândia, Dinamarca, França, entre outros (VIRKUS, 2003).

A necessidade de mais ações para o desenvolvimento da competência em informação é destacada por Minsenti (2004). O autor relata que dentro das universidades está a maior parte das pesquisas sobre o tema e refere-se a dados de análises divulgadas pelo Centro Studi Investimenti Sociali ${ }^{8}$ (CENSIS) (MINSENTI, 2004). Essas análises indicavam que apenas 16\% dos cidadãos italianos eram capazes de dominar os recursos digitais e que, ainda assim, em 2003 apenas $15 \%$ das bibliotecas italianas organizavam cursos para os usuários de forma sistemática. Nessa perspectiva, Minsenti (2004) afirma a necessidade de repensar os cursos para o desenvolvimento da competência em informação, uma vez que 
[...] a information literacy não pretende mais limitar-se a ensinar a usar a biblioteca e as técnicas de pesquisa bibliográfica. Na era digital é necessário perceber que os recursos de informação estão também, e às vezes, especialmente, fora dos limites da biblioteca [...] Finalmente, a information literacy é uma oportunidade para repensar o papel profissional do bibliotecário e da organização do trabalho na biblioteca. (MINSENTI, 2004, p. 1-2, grifo nosso, tradução nossa).

Sob este foco, diferentes formas de desenvolver a competência em informação passam a ser apresentadas na Itália, como a pesquisa-ação, método aplicado por Vezzosi (2006) no contexto universitário, em pesquisa realizada na Universidade de Parma. A autora conclui que essa metodologia pode promover o compartilhamento de ideias e pode ser utilizada para viabilizar mudanças efetivas em atividades para o desenvolvimento da competência em informação ao unir a pesquisa com a prática (VEZZOSI, 2006).

Ainda em bibliotecas universitárias, há a avaliação apresentada por Piccinini (2006) sobre o grau de competência em informação dos usuários da Biblioteca Universitaria di Lugano. Os resultados mostraram que os estudantes estão conscientes da importância da competência em informação e que é desejável um maior envolvimento da biblioteca e dos professores em prol de programas nesse sentido (PICCININI, 2006).

Zani (2007) citou a importância dos Information Literacy Competency Standards for Higher Education, publicados pela Association of College \& Research Libraries (ACRL). Para o autor, o documento foi relevante por influenciar as reflexões acerca das atividades dos bibliotecários de todo o mundo (ZANI, 2007). O autor ainda destaca que a temática não deve ser preocupação apenas das pessoas comuns, mas de todos os agentes institucionais envolvidos na formação básica das pessoas em todos os níveis de ensino (ZANI, 2007).

A biblioteca escolar é foco de Das (2007). O autor aborda a necessidade de adaptação dos bibliotecários escolares para desenvolverem ações voltadas a um estudante conectado ao mundo digital (DAS, 2007). O autor acrescenta que no cenário europeu não há muitos dados sobre as bibliotecas escolares ou programas específicos de formação dos seus bibliotecários e propõe a cooperação entre as bibliotecas escolares e as bibliotecas públicas (DAS, 2007). 
Em 2009, ocorreu o Spring Event - evento anual para reforçar a ligação entre bibliotecários italianos e estadunidenses, por meio de debates e trocas de experiências - com a presença de Woody Horton Jr. e Carla Basili, especialistas em competência em informação (CAMPOS CERVERA, 2009). Ballestra (2009) destaca a seção sobre competência em informação da IFLA, criada em 2002, a qual visa “[...] promover a difusão de ações de Information Literacy em todos os tipos de bibliotecas [...]" (BALLESTRA, 2009, p. 4, tradução nossa). O termo information literacy é traduzido por Ballestra (2009) como "alfabetizzazione all'informazione".

Em 2009, novas tendências se apresentam ao tema: o Congresso da IFLA aconteceu em Milão e abordou a necessidade de maior flexibilidade para quando os bibliotecários forem tratar do desenvolvimento da competência em informação, uma vez que os caminhos são diferentes de acordo com os usuários. Por esse motivo, há maior necessidade de os bibliotecários dessas bibliotecas programarem ações práticas de competência em informação conforme os diferentes tipos de usuários (BALLESTRA, 2009).

$\mathrm{Na}$ vertente tecnológica, Fiorentini (2010) estuda as relações entre a competência em informação e a Web 2.0 e conclui que, em geral na Itália, prevalecem os meios tradicionais para o desenvolvimento dessa competência por faltarem iniciativas para o uso adequado da Web 2.0 neste processo, limitado aos documentos de texto que o usuário pode consultar.

$\mathrm{Na}$ via de estabelecer novas possibilidades, a temática passa a ser explorada em publicações com diferentes focos, como a TreeLLLe ${ }^{9}$, que trata da educação de adultos na Itália e Europa. Nesta publicação, há o conceito de competência em informação como a "[...] capacidade de gerir e avaliar criticamente o fluxo crescente de informações e imagens aos quais estamos imersos nos novos meios de comunicação de massa." (TREELLLE, 2010, p. 198, tradução nossa). Há também a consciência de que a competência em informação "[...] ajuda os cidadãos adultos a distinguir as fontes, a separar os fatos das opiniões, as verdades comprovadas das ficções e dos rumores." (TREELLLE, 2010, p. 199, tradução nossa). O programa da TreeLLLe propõe a competência em informação como um filtro crítico, como um instrumento 
difundido nas comunicações de massa. Além disso, no mesmo programa se indica a necessidade de desenvolver a competência em informação na educação de base junto a programas especiais dedicados aos adultos, para que assim a população seja autônoma e tenha espírito crítico para trabalhar com os meios de comunicação de massa.

Eventos também mostram a relevância da competência em informação na Itália: um destes é o European Meeting on Media and Information Literacy Education in Libraries (and beyond) (EMMILE). Ocorrido em Milão no ano de 2012, foi uma conferência de três dias que uniu diversas entidades para explorar aspectos teóricos e práticos da temática (EUROPEAN MEETING ON MEDIA AND INFORMATION LITERACY EDUCATION IN LIBRARIES (AND BEYOND), 2012). O evento teve como idioma oficial o inglês, mas contou com tradução simultânea para 0 italiano. Estiveram presentes importantes pesquisadores da área como Carla Basili, Laura Ballestra e Luisa Marquardt (EUROPEAN MEETING ON MEDIA AND INFORMATION LITERACY EDUCATION IN LIBRARIES (AND BEYOND), 2012). Em 2012, em Pádua, ocorreu o Seminário da Associação Italiana de Bibliotecas (AIB), evento com debates significativos (BASILI, 2014). Nesse seminário, houve o evento paralelo intitulado Information literacy: teorie e buone pratiche $e^{10}$, que contou com palestras de Laura Ballestra, Carla Basili e outros pesquisadores.

A conexão entre a competência em informação e a tecnologia é visível nos estudos italianos. Pennazio, Traverso e Parmigiani (2013) afirmam que a competência em informação se refere “[...] à capacidade de pesquisar adequadamente para obter informações utilizando os instrumentos disponíveis [...]" (PENNAZIO; TRAVERSO; PARMIGIANI, 2013, p. 36, tradução nossa). Na pesquisa dos autores, a competência em informação é necessária para atingir a competência digital (PENNAZIO; TRAVERSO; PARMIGIANI, 2013).

A partir de 2013, passou a ocorrer a European Conference on Information Literacy. Nesta primeira edição, o evento ocorreu em Istambul, na Turquia, e seu comitê permanente de organização contou com mais de 130 membros, de diversos países, inclusive de pesquisadores de instituições italianas (EUROPEAN CONFERENCE ON INFORMATION LITERACY, 2013), foram 
eles: Piero Cavaleri e Laura Ballestra, que trataram da formulação do foco adotado pelos indivíduos nos processos de pesquisa acadêmica; Licia Sbattella, Roberto Tedesco e Silvia Sbattella, com a abordagem da competência em informação para estudantes universitários com deficiência; Elena Collina, Massimo Urbini, Alina Renditiso e Fabio Zauli, com a proposta de diretrizes para serem usadas como guia nos programas de competência em informação das mais de cem bibliotecas da Università di Bologna; as italianas Paola de Castro e Daniela Marsili junto com os turcos Serap Kurbanoğlu e Tezer Kutluk, que ofereceram um workshop para tratar da competência em informação na área da saúde de modo a propor o desenvolvimento de programas com esta temática, dos quais participassem bibliotecários e profissionais da área da saúde, e Aldo Pirola, que coordenou um painel com os holandeses Vincent Bonnet e Marian Koren, no painel houve debates sobre o papel das bibliotecas na sociedade do conhecimento, refletindo como estas instituições podem se envolver nas políticas nacionais.

O ECIL de 2014 foi realizado na Croácia e apresentou as seguintes pesquisas desenvolvidas na Itália: Carla Basili e Stéphane Goldstein publicaram um artigo acerca da conscientização de políticas sobre a competência em informação científica na pesquisa. Caroline Venaille apresentou sua pesquisa sobre intercompreensão em notícias online em busca da ética no jornalismo (EUROPEAN CONFERENCE ON INFORMATION LITERACY, 2014). Elena Collina, Alina Renditiso e Fabio Zauli destacaram o papel do bibliotecário no desenvolvimento da competência em informação de estudantes universitários e apresentaram o acompanhamento de sua pesquisa prática com bibliotecários da Università di Bologna (EUROPEAN CONFERENCE ON INFORMATION LITERACY, 2014). Por fim, Daniela Marsili, Pietro Comba e Paola de Castro apresentaram um pôster sobre competência em informação na área da saúde para alertar sobre exposições perigosas, como o caso do amianto e doenças relacionadas (EUROPEAN CONFERENCE ON INFORMATION LITERACY, 2014).

No ano de 2015, o país sede da ECIL foi a Estônia e as pesquisas italianas apresentadas foram as seguintes: Simonetta Pasqualis Dell'Antonio, 
Siri Nangah Spora e Gabriella Taddeo trataram de projetos de competência em informação com imigrantes na Itália; Elena Collina, Alina Renditiso e Fabio Zauli exibiram um projeto de competência em informação desenvolvido com discentes da Università di Bologna e coordenado por bibliotecários, o curso foi ofertado aos 15 mil alunos da instituição (EUROPEAN CONFERENCE ON INFORMATION LITERACY, 2015); Christine Furno, Madeline Mundt, Livia Piotto, Michael Stoepel e Tatevik Zargaryan apresentaram pôster acerca da sua pesquisa em andamento com um grupo focal formado com professores e bibliotecários do AMICAL Consortium ${ }^{11}$ sobre competência em informação, e o pôster de Valentina Rovacchi e Gabriella Tufano abordou a criação de um wiki ${ }^{12}$ para divulgar projetos de competência em informação desenvolvidos em bibliotecas acadêmicas da Itália (EUROPEAN CONFERENCE ON INFORMATION LITERACY, 2015).

Em 2016, a ECIL ocorreu em Praga, na República Tcheca, e foram apresentados três trabalhos desenvolvidos na Itália: Luisa Marquardt publicou artigo com os resultados de um estudo comparativo no sul da Itália, que concluiu que a existência da biblioteca escolar impacta a educação e os processos de desenvolvimento da competência em informação dos discentes (EUROPEAN CONFERENCE ON INFORMATION LITERACY, 2016). Paola de Castro, Cristina Agresti, Elena Ambrosini, Maria Cristina Barbaro e Sandra Salinetti apresentaram projeto-piloto sobre competência em informação na área da saúde nas escolas e o desenvolvimento de consciência sobre a saúde do planeta, e o artigo de Laura Ballestra e Piero Cavaleri apresenta estudo em andamento a respeito da difusão de atividades de educação financeira em bibliotecas públicas italianas e questiona se cursos de competência em informação podem potencializar essas atividades (EUROPEAN CONFERENCE ON INFORMATION LITERACY, 2016).

As pesquisas italianas apresentadas na ECIL de 2017, na cidade de SaintMalo, na França, foram três: Paola De Castro abordou a contribuição necessária dos pesquisadores para com a sociedade, a partir da comunicação de suas pesquisas fora da comunidade acadêmica (EUROPEAN CONFERENCE ON INFORMATION LITERACY, 2017). Elena Collina e Rita Patregnani 
apresentaram pesquisa sobre as possíveis lacunas no gerenciamente de dados e as possibilidades que a competência em informação pode trazer aos bibliotecários, e Michael Stoepel, Tatevik Zargaryan, Livia Piotto, Christine Furno e Krasimir Spasov relataram os resultados de um workshop sobre a integração da competência em informação e os cursos superiores, ocorrido na França (EUROPEAN CONFERENCE ON INFORMATION LITERACY, 2017).

A edição da ECIL de 2018, ocorreu em Oulu, na Finlândia (EUROPEAN CONFERENCE ON INFORMATION LITERACY, 2018). Com reduzido número de apresentações, foram identificadas as pesquisas italianas de Marina Encheva, Plamena Zlatkova, Anna Maria Tammaro e Mats Brenner, apresentando o Erasmus + project NAVIGATE - Information Literacy, projeto que baseia o aprendizado em jogos a fim de trabalhar como lidar com os conteúdos falsos disponíveis, com parceria entre instituições da Bulgária, Itália e Suécia (EUROPEAN CONFERENCE ON INFORMATION LITERACY, 2018).

Na Itália existe o Gruppo di Studio Nazionale AIB sulla Information Literacy $^{13}$ (GLIT), vinculado à AIB. Para o GLIT (2016), a competência em informação possui raízes nas ações de seleção de informação, referência e educação para informação. Para o grupo, enquanto associação de profissionais de bibliotecas, é necessária uma reflexão que coloca o direito à informação e aos serviços de informação no núcleo das bibliotecas. Para este fim, o GLIT busca uma definição complementar à da IFLA que reflita as peculiaridades da Itália e da Europa. Assim, o grupo publicou em 2016 o Manifesto per l'information literacy $^{14}$, sugerindo possibilidades e oportunidades para ações de formação. Este documento mostra que a competência em informação pode ser eficiente para lidar com o analfabetismo funcional - incapacidade de compreender de modo crítico a informação. A relevância social também é abordada, uma vez que, para o GLIT (2016, p. 3), “As habilidades em informação desempenham um papel importante na vida cotidiana, influenciando o estado de saúde, a consciência política, a participação ativa e consciente da vida pública.” (GLIT, 2016, doc. não paginado, tradução nossa). No Manifesto do GLIT (2016) é 
exposta uma série de esquemas com finalidade de propor dezesseis temas a fim de gerar iniciativas para o desenvolvimento da competência em informação:

Figura 1 - Iniciativas para a competência em informação, de acordo com o Manifesto per l'information literacy de 2016

Fonte: Adaptado de GLIT (2016).

\begin{tabular}{|c|c|c|}
\hline $\begin{array}{l}\text { Utilização dos mecanismos de } \\
\text { pesquisa para obter informações }\end{array}$ & & $\begin{array}{c}\text { Utilização da Wikipédia para obter } \\
\text { informações }\end{array}$ \\
\hline $\begin{array}{l}\text { Gerenciamento de modo consciente } \\
\text { da identidade e informações pessoais } \\
\text { on-line }\end{array}$ & & $\begin{array}{l}\text { Utilização das fontes abertas para obter } \\
\text { informações }\end{array}$ \\
\hline $\begin{array}{c}\text { Expressar-se na rede de forma ética e } \\
\text { responsável }\end{array}$ & & $\begin{array}{l}\text { Utilização das fontes especializadas } \\
\text { para obter informações }\end{array}$ \\
\hline $\begin{array}{l}\text { Ecossistema contemporâneo de } \\
\text { informação }\end{array}$ & Iniciativas para a & Avaliação crítica da informação \\
\hline $\begin{array}{c}\text { Organização semântica nas } \\
\text { bibliotecas }\end{array}$ & $\begin{array}{l}\text { Competência em } \\
\text { Informação }\end{array}$ & $\begin{array}{l}\text { Utilização da informação de modo } \\
\text { ético }\end{array}$ \\
\hline $\begin{array}{l}\text { Utilização de modo consciente dos } \\
\text { dispositivos móveis e aplicativos }\end{array}$ & & $\begin{array}{l}\text { Prevenção e gerenciamento da } \\
\text { sobrecarga de informação }\end{array}$ \\
\hline $\begin{array}{c}\text { Aperfeiçoamento do impacto da } \\
\text { comunicação cientifica produzida } \\
\text { como autor } \\
\text { Conhecimento das fontes de } \\
\text { informação apropriadas para a própria } \\
\text { área }\end{array}$ & & $\begin{array}{l}\text { Pesquisa documental como pesquisa } \\
\text { estruturada } \\
\text { Compartilhamento e criação de modo } \\
\text { colaborativo e eficaz conteúdos e } \\
\text { informações }\end{array}$ \\
\hline
\end{tabular}

Na tendência de desenvolver a competência em informação nas pessoas, Paiano (2016) aponta outro aspecto relevante dos programas, a possibilidade de capacitá-las para o mundo do trabalho. O autor afirma que consequências positivas de trabalhar o desenvolvimento da competência em informação no mundo do trabalho são o aumento da eficiência, a redução de custos, a gestão do conhecimento mais eficiente, a superação da sobrecarga de informação, uma maior capacidade de resolver problemas, uma melhor comunicação corporativa, entre outros aspectos (PAIANO, 2016).

A questão tecnológica é apresentada na pesquisa de Catalani (2017), quando o autor se preocupou em analisar a colaboração entre bibliotecas e Fundação Wikimedia ${ }^{15}$. Para o autor, a Wikipédia é um instrumento em 
potencial para auxiliar no desenvolvimento da competência em informação (CATALANI, 2017).

Com vistas a sintetizar as vertentes das temáticas da competência em informação estudadas na Itália e que se fizeram presentes no estudo bibliográfico realizado para os fins deste trabalho, a figura a seguir ilustra tais vertentes, sugerindo um olhar atento de investigadores interessados no que se tem estudado na área no contexto italiano.

Figura 2 - Temáticas abordadas sobre competência em informação na Itália

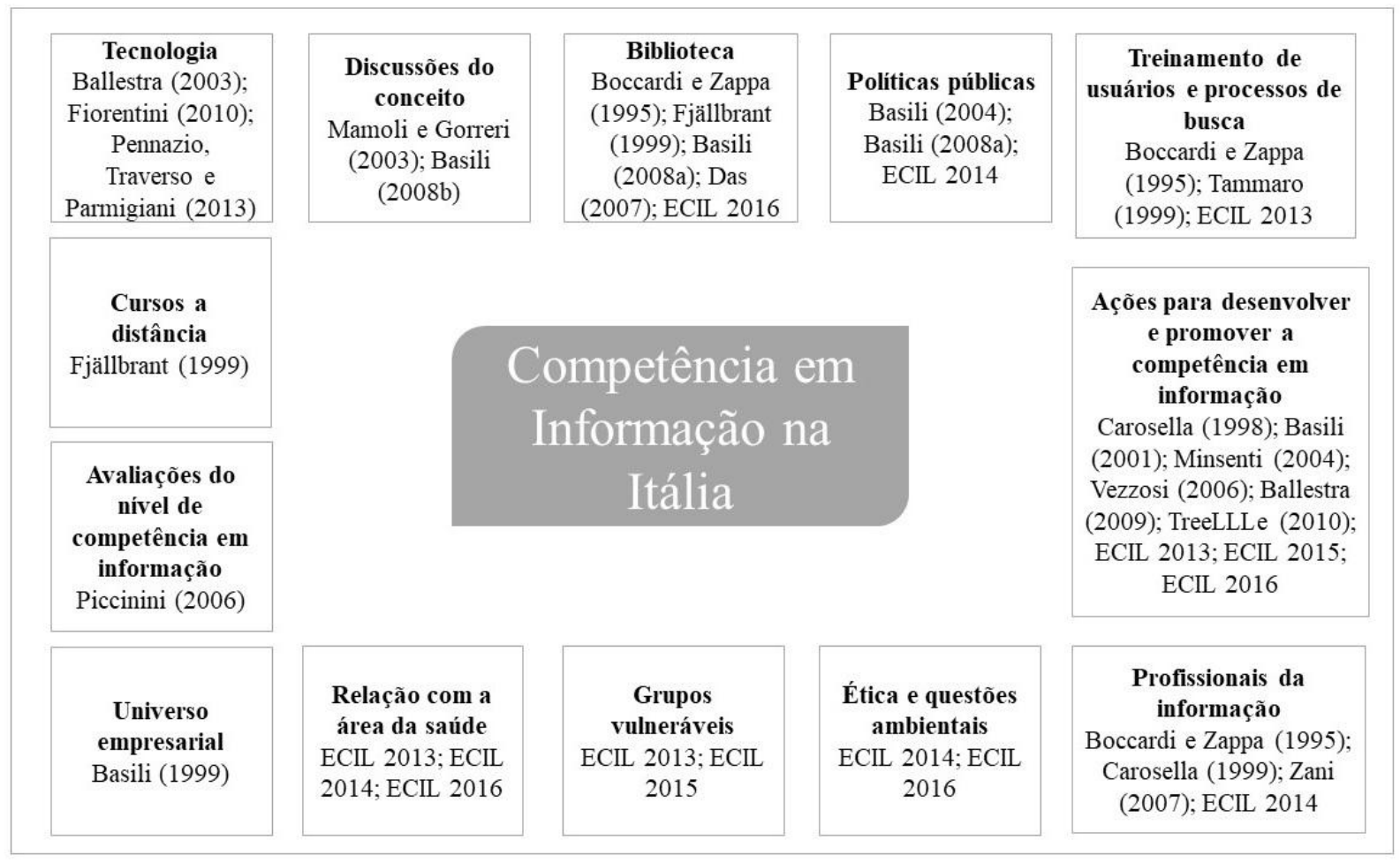

Fonte: Dados da pesquisa.

O desenvolvimento da competência em informação tem se mostrado relevante para os pesquisadores italianos. O reconhecimento das próprias necessidades de informação e a capacidade de localizar, recuperar, avaliar, organizar e utilizar as informações são essenciais para a formação de cidadãos conscientes na realidade italiana. Neste sentido, Carla Basili lidera os estudos na área nesse país, sendo também relevantes publicações de Laura Ballestra, Maria Pia Carosella e Alina Renditiso. Dois dos primeiros documentos mais importantes para o tema são de 1998 e de 2001. A primeira é de Carosella e 
disserta sobre o desenvolvimento da competência em informação em solo europeu. A segunda traz os debates da information literacy para a Itália; ela se intitula La società dell'informazione: "information literacy”: un concetto solo statunitense? e foi publicada por Basili.

Observou-se que as pesquisas iniciais sobre competência em informação na Itália apontam a necessidade de trabalhar uma certificação para os profissionais da informação, de acordo com suas capacidades, tratando também do tema em empresas privadas. Os estudos versam sobre a formação de usuários no ensino a distância; analisam como as bibliotecas - principalmente as universitárias - lidam com o assunto; propõem projetos para desenvolvimento de estratégias europeias pela competência em informação; mostram preocupação com os meios utilizados para o seu desenvolvimento; ressaltam a sua importância para a educação de adultos e demonstram as suas relações com a tecnologia e a necessidade de políticas públicas para a institucionalização da competência em informação. Além disso, discutiu-se sobre o conceito de competência de informação, foram organizados diversos eventos da área e formaram-se grupos de estudos.

\section{Considerações Finais}

Neste artigo foram apresentados o histórico da competência em informação, definições do conceito, artigos resultantes do levantamento sobre os estudos do tema na Itália, os procedimentos de pesquisa e seus resultados.

Considera-se que este trabalho alcançou o objetivo proposto ao apresentar ao leitor os documentos já publicados sobre competência em informação na Itália. O primeiro objetivo específico foi atendido, ao apontaremse as fontes de informação sobre o tema. Neste sentido, a base de dados DOAJ, dado que seu número de resultados é maior, pode ser considerada, com o levantamento de Basili (2014), uma das melhores fontes. As revistas científicas italianas da área da Biblioteconomia e da Ciência da Informação são a principal fonte para publicações de estudos sobre o tema. Os contextos abordados são diversos e apresentam melhorias no que diz respeito ao desenvolvimento de ações práticas para o desenvolvimento da competência em informação na Itália. 
O segundo objetivo foi identificar as principais publicações sobre o tema da pesquisa. Foi alcançado, com a exposição do referencial teórico, na seção 3 deste artigo, com destaque a autoras como Carla Basili, Laura Ballestra, Maria Pia Carosella e Alina Renditiso.

A partir dos dados desta pesquisa, identificou-se que os pesquisadores da Itália se mostram preocupados com o desenvolvimento da competência em informação e com sua importância em diversas situações, como no mercado de trabalho, nas universidades e na formação dos profissionais.

O surgimento de eventos de grande porte, como a ECIL, e que movimentam um número considerável de pesquisadores europeus, faz da competência em informação um tema cada vez mais relevante no cenário da Ciência da Informação. Observa-se, por meio do aumento do número de documentos publicados e pesquisas sobre a temática na Itália, que os pesquisadores desse país vêm aprofundando as pesquisas e buscando a promoção de ações práticas para o desenvolvimento da competência em informação nos indivíduos. De acordo com Virkus (2013), dos 31 países europeus relacionados e dos 1.252 documentos levantados pelo autor com buscas na base de dados Web of Science, a Itália é o décimo segundo país com mais publicações sobre o tema no período de 2003 a 2013.

Com os documentos recuperados nesta pesquisa, foi possível atender ao terceiro objetivo específico, ou seja, identificar as temáticas apresentadas nos documentos publicados. Assim, foram identificados estudos italianos relacionando a competência em informação com: tecnologia; aspectos voltados às bibliotecas; preocupação com as políticas públicas inexistentes ou insuficientes; treinamento de usuários e processos de busca de informação; ações práticas; relações com os profisssionais da informação; questões éticas e ambientais; grupos vulneráveis - como imigrantes e deficientes; a área da saúde; o universo empresarial, entre outros elementos.

Quando o GLIT (2016) se propõe a refletir acerca de temas que possam gerar iniciativas relevantes para a competência em informação, dá destaque também à questão da sobrecarga de informação e destaca a necessidade de prevenir e gerenciar essa sobrecarga. Para trabalhar essa questão dentro de 
programas de competência em informação, o grupo sugere a utilização de ferramentas e técnicas para filtrar o fluxo de informações online, considerando que a exposição ao excesso de informação pode gerar sobrecarga, capaz de acarretar declínio da capacidade de selecionar de modo eficaz as informações.

Nesta perspectiva, pretende-se investigar em trabalhos futuros a ideia da sobrecarga de informação e a interconexão disso com a competência em informação, no sentido de minimizar danos causados ao ser humano.

\section{Referências}

BALLESTRA, Laura. E-learning e information literacy: un connubio vincente. Biblioteche Oggi, Milano, v. 10, n. 21, p. 11-23, 2003.

BALLESTRA, Laura. Information literacy. In: INTERNATIONAL FEDERATION OF LIBRARY ASSOCIATIONS GENERAL CONFERENCE AND ASSEMBLY, 75., 2009, Milano. Apresentação, artigos, palestras, instruções.... Associazione Italiana Biblioteche Notizie, Roma, v. 21, n. 6, 2009.

BASILI, Carla. Information and education policies in Europe: key factors influencing information literacy academic policies in Europe. In: BASILI, Carla (ed.). Information Literacy at the crossroad of education and information policies in Europe. Roma: Consiglio Nazionale Delle Ricerche, 2008a. p. 1832.

BASILI, Carla. Theorems of information literacy. In: BASILI, Carla (ed.). Information Literacy at the crossroad of education and information policies in Europe. Roma: Consiglio Nazionale Delle Ricerche, 2008b. p. 33-52.

BASILI, Carla. La cultura dell'informazione: opportunità e strategie. IDAInformazioni, [s.l.], v. 17, n. 2, p. 11-12, 1999.

BASILI, Carla. "Information literacy": un concetto solo statunitense? AIDAInformazioni, [s.l.], v. 19, n. 2, p. 10-15, 2001.

BASILI, Carla. List of selected Information Literacy Resources available in the Italian language. In: HORTON JUNIOR, Forest Woody. Overview of information literacy resources worldwide: "helping people to easily and quickly find the information they need". 2nd. ed. Paris: UNESCO, 2014. p. 375384.

BASILI, Carla. Verso una patente europea dell'informazione: il progetto EnIL (European network on Information Literacy). Biblioteche Oggi, Milano, v. 5, n. 22, p. 45-51, 2004. 
BOCCARDI, Donata; ZAPPA, Roberto. Percorsi di ricerca e autonomia dello studente. Biblioteche Oggi, Milano, v. 13, n. 10, p. 54-62, 1995.

CAMPOS CERVERA, Gimena. Spring event 2009: uno sguardo all' information literacy tra l'italia e gli stati uniti. Associazione Italiana Biblioteche Notizie, Roma, v. 21, n. 5, p. 25-25, 2009.

CAREGNATO, Sônia Elisa. O desenvolvimento de habilidades informacionais: o papel das bibliotecas universitárias no contexto da informação digital em rede. Revista de Biblioteconomia e Comunicação, Porto Alegre, v. 8, p. 47-55, 2000 .

CAROSELLA, Maria Pia. DECIDoc: develop european competencies in information and documentation. AIDAInformazioni, [s. l.], v. 17, n. 3-4, p 1-2, 1999.

CAROSELLA, Maria Pia. DECID: sviluppo di competenze di informazione e documentazione a livello europeo. AIDAInformazioni, [s. l.], v. 16, n. 1, p. 20, 1998.

CATALANI, Luigi. Biblioteche e Wikimedia: strategie comuni per l'accesso aperto alla conoscenza e la costruzione collaborativa del sapere libero. JLIS.it, Firenze, v. 8, n. 3, p. 100-114, 2017.

CROCETTI, Luigi (org.). Information literacy per ragazzi: come favorire la competenza informativa. [S. l.: s. n.], 2012.

DAS, Lourense. Molto più di una biblioteca...: ruolo e funzioni della biblioteca scolastica di fronte al mutare dei processi di apprendimento: una prospettiva internazionale. Sfoglialibro, Milano, p. 3-9, 2007.

EUROPEAN CONFERENCE ON INFORMATION LITERACY, 1., 2013, Istanbul. Proceedings [...]. Instabul: Hacettepe University, 2013.

EUROPEAN CONFERENCE ON INFORMATION LITERACY, 2., 2014, Dubrovnik. Proceedings [...]. Dubrovnik: University of Zagreb, 2013.

EUROPEAN CONFERENCE ON INFORMATION LITERACY, 3., 2015, Tallinn. Proceedings [...]. Tallinn: Tallinn University, 2015.

EUROPEAN CONFERENCE ON INFORMATION LITERACY, 4., 2016, Prague. Proceedings [...]. Prague: Association of Libraries of Czech Universities, 2016.

EUROPEAN CONFERENCE ON INFORMATION LITERACY, 5., 2017, Saint Malo. Proceedings [...]. Saint Malo: Information Literacy Association, 2017. 
EUROPEAN CONFERENCE ON INFORMATION LITERACY, 6., 2018, Oulu. Proceedings [...]. Oulu: University of Oulu, 2018.

\section{EUROPEAN MEETING ON MEDIA AND INFORMATION LITERACY EDUCATION IN LIBRARIES (AND BEYOND), 2012, Milano. Proceedings [...]. Milano: [s. n.], 2012.}

FIORENTINI, Barbara. Quando l'information literacy incontra il web 2.0: per la formazione dell'utente il bibliotecario può oggi disporre di efficaci strumenti di comunicazione e condivisione. Biblioteche Oggi, Milano, v. 28, n. 10, p. 39-46, 2010 .

FJÄLLBRANT, Nancy. Biblioteche e apprendimento a distanza: alcuni progetti europei nell'ambito della information literacy. Biblioteche Oggi, Milano, v. 17, n. 10, p. 54-59, 1999.

GLIT (Itália). Manifesto per l'Information Literacy. [S. l.]: AIB-WEB, 20 nov. 2016.

HORTON JUNIOR, Forest Woody. Overview of information literacy resources worldwide: "helping people to easily and quickly find the information they need". 2nd. ed. Paris: UNESCO, 2014.

\section{LAU, Jesús. Diretrizes sobre desenvolvimento de habilidades em} informação para a aprendizagem permanente. The Hague: IFLA, 2007.

MAMOLI, Fiammetta; GORRERI, Marina. Dalla formazione dei bibliotecari alla formazione degli studenti: un progetto dell'Università di Parma.

Biblioteche Oggi, Milano, v. 21, n. 4, p. 21-28, 2003.

MINSENTI, Pierfranco. Information literacy al salone di Torino. Associazione Italiana Biblioteche Notizie, Roma, v. 16, n. 8, p. 15-16, 2004.

PAIANO, Tommaso. Information literacy e mondo del lavoro: un connubio possibile anche in Italia? Associazione Italiana Biblioteche Studi, Roma, v. 56, n. 2, p. 241-264, 2016.

PENNAZIO, Valentina; TRAVERSO, Andrea; PARMIGIANI, Davide. Digital literacies a scuola: i profili digitali degli studenti. Td Tecnologie Didattiche, $[s$. l.], v. 21, n. 1, p. 35-40, 2013.

PICCININI, Sabrina. Valutare il grado di comprensione delle information literacy skills da parte degli studenti universitari: dall'esperienza della biblioteca universitaria di Lugano una riflessione sulle tecniche di ricerca. Biblioteche Oggi, Milano, v. 24, n. 3, p. 17-27, 2006. 
RENDITISO, Alina. La sezione IFLA sulla competenza informativa al congresso di Buenos Aires 2004. Bibliotime, Bologna, v. 7, n. 3, p. 01-06, 2004.

TAMMARO, Anna Maria. Apprendere ad apprendere: la ricerca fai-da-te. Biblioteche Oggi, Milano, v. 17, n. 10, p. 46-52, 1999.

TOMAÉL, Maria Inês; ALCARÁ, Adriana Rosecler; SILVA, Terezinha Elisabeth da. Fontes de informação digital: critérios de qualidade. In: TOMAÉL, Maria Inês; ALCARÁ, Adriana Rosecler (org.). Fontes de informação digital. Londrina: Eduel, 2016. cap. 1.

TREELLLE. Consumi culturali, indici di lettura, uso dei media: l'importanza della information literacy. In: TREELLLE. Il lifelong learning e l'educazione degli adulti in Italia e in Europa Dati confronti e proposte. 9th. ed. Genova: Associazione Treellle, 2010. cap. 4.

VEZZOSI, Monica. Information literacy and action research: an overview and some reflections. New Library World, [s. l.], v. 107, n. 7-8, p. 286-301, 2006.

VIRKUS, Sirje. Information literacy in Europe: a literature review. Information Research, [s. l.], v. 8, n. 4, p. 1-90, 2003.

VIRKUS, Sirje. Information literacy in Europe: ten years later. Tallinn: Tallinn University, 2013.

ZANI, Maurizio. Le competenze informative degli studenti di scienze e ingegneria. Bibliotime, Bologna, v. 10, n. 1, 2007.

\title{
Information Literacy: introductory studies on the subject in
}

\section{Italy}

\begin{abstract}
This study addresses the issue of information literacy in Italy. With the increasing amount of information available to the general public, the development of information literacy has also risen in importance in contemporary society. Through bibliographic research, this article reviews the published literature about information literacy in Italy. It points to information sources featuring available work on the subject, as well as the main publications dealing with it, and provides a survey of the topics discussed in the scientific literature about information literacy in Italy. Studies were retrieved from the Directory of Open Access Journals, Library and Information Science Abstracts, Information Science \& Technology Abstracts and Web of Science databases and from surveys published by Basili (2014) and Crocetti (2012). Data gathered from 2013 to 2018 from the European Conferences on Information Literacy
\end{abstract}


complemented those results, and a total of 136 documents was identified. Discussions about information literacy in Italy were found to have been growing since 1990 and to have intensified after 2004 with the work of influent Italian researchers such as Carla Basili and Laura Ballestra. The Italian studies reviewed were found to relate information literacy to several topics, among which: concerns about nonexistent or insufficient public policy, practical actions, ethical and environmental issues, vulnerable groups, the health care field and technology.

Keywords: Information literacy. Literature review. Italy.

Recebido: $28 / 10 / 2019$

Aceito: 20/04/2020

\section{Como citar:}

SOUZA, Marcela Reinhardt de; VITORINO, Elizete Vieira. Competência em Informação: estudos introdutórios sobre a temática na Itália. Em Questão, Porto Alegre, v. 26, n. 3, p. 354-379, set./dez. 2020. DOI:

https://doi.org/10.19132/1808-5245263.354-379.

1 Algumas traduções, descritas por Horton Junior (2014), são: “maitrise de l'information" em francês; "informationskompetenz" em alemão; "competenza informativa" em italiano; "alfabetización informacional" ou "competencias informacionales" em espanhol.

${ }^{2} \mathrm{Na}$ tradução para o português: Information literacy para os jovens: como promover a competência em informação.

${ }^{3} \mathrm{Na}$ tradução para o português: AIDA informações: revista de Ciência da Informação.

${ }^{4} \mathrm{Na}$ tradução para o português: Guia europeu de referência de informação e documentação para os profissionais da informação e documentação.

${ }^{5} \mathrm{Na}$ tradução para o português: A cultura da informação: oportunidade e estratégia.

${ }^{6} \mathrm{Na}$ tradução para o português: alfabetização para o uso das informações.

${ }^{7} \mathrm{Na}$ tradução para o português: cultura da informação.

${ }^{8} \mathrm{Na}$ tradução para o português: Centro de Estudos de Investimentos Sociais.

${ }^{9}$ Associação sem fins lucrativos com o objetivo de melhorar a qualidade na educação.

${ }^{10} \mathrm{Na}$ tradução para o português: Information literacy: teorias e boas práticas.

${ }^{11}$ Consórcio que reúne 27 instituições de Ensino Superior em prol de promover o aprendizado, o ensino e a pesquisa com serviços colaborativos de bibliotecas e informações e recursos curriculares nas instituições membros.

${ }^{12}$ Software colaborativo.

${ }^{13} \mathrm{Na}$ tradução para o português: Grupo de Estudos Nacional AIB sobre competência em informação.

${ }^{14} \mathrm{Na}$ tradução para o português: Manifesto pela competência em informação.

${ }^{15}$ Fundação sem fins lucrativos criada em 2003 que "[...] objetiva incentivar o desenvolvimento e a disseminação de conteúdo multilíngue gratuito e fornecer gratuitamente a todo o público 
conteúdo de seus projetos wiki, entre os quais o mais conhecido é a Wikipédia." (CATALANI, 2017 , p. 101, grifo nosso, tradução nossa). 